\title{
New Books - Livres nouveaux - Buchbesprechungen
}

J. Delmas et G. Laux: Système nerveux sympathique. Etude systématique et macroscopique, Introduction à la physiopatbologie du Sympathique. Masson et Cie., Paris 1952. 337 Seiten, 10 Tafeln, 146 Abb., ffrs. 4200.-.

Nach einleítenden Kapiteln über die Gliederung, die Entwicklung, die mikroskopisclie Anatomie und den Mechanismus der Impulsübermittlung im autonomen System werden seine Zentren und Bahnen in den Zentralorganen be-schrieben und zwar für das Rückenmark, den Bulbus und das Zwischenhim in Anlelinung an die grundlegenden Arbeiten Laruelles. Auch die Beziehungen der Endhirnrinde zu den autonom innervierten Organen und ihre praktischen Kon-sequenzen sind berücksichtigt. Dann folgt das Grundsätzliche über den Faserverlauf im Ortho- und Parasympathicus mit Betrachtungen über Reflexe aller Art und über Schmerzleitung. Das größte Kapitel umfaßt die makroskopische Beschreibung der autonomen Nerven und zwar zunächst der sympathischen (Grenzstrang, präverte-brale Ganglien, Rami communicantes, viscerale Äste), dann der parasympathischen (kranialer und sakraler Teil). In besonderen Kapiteln werden die autonomen Nerven der Eingeweide, der Sinnesorgane und der endokrinen Drüsen dargestellt, und den Schluß bildet ein ausführliches Kapitel über die Innervation der Gefäße.

In den Schlußfolgerungen wird das autonome System als ein „chapitre ingrat” der Anatomie bezeichnet. Kein Einsichtiger wird diesem Satze nicht zu-stimmen. Denn auf keinem anderen Gebiete der Anatomie gehen selbst die grund-sätzhchsten Anschauungen so weit auseinander. Der eine wird die Existenz trophischer Nerven bezweifeln, ein anderer den spinalen Parasympathicus im Sinne von Ken Kuré, ein Dritter die von den Spinalganglien gegebene Beschreibung, und die Gegner der Neurenlehre werden ihre besonderen Vorbehalte anbringen. Aber wie immer sich der Einzelne zur Problematik des autonomen Systems verhalten mag, verdient das Werk von Delmas und Laux allgemeine Beachtung. Denn es ist das Er-gebnis intensiver und jahrzehntelanger persönlicher Bemühungen seiner Verfasser und ihrer zahlreichen, der Anatomenschule von Montpellier würdigen Mitarbeiter. Dafür zeugt nicht zuletzt das Literaturverzeichnis, denn es enthält nicht weniger als 118 Arbeiten aus dem anatomischen Laboratorium von Montpellier. Aber auch die übrige Literatur ist ausgiebig berücksichtigt, insbesondere die französische, der wir ja die Erschließung wichtiger Gebiete der autonomen Innervation verdanken.

E. Ludwig, Basel

News from Universities - Nouvelles universitaires Universitätsnachrichten Germany - Allemagne - Deutschland Erlangen: Dr. Helmut Leonhardt hat sich für Anatomie habilitiert. Mainz: Dr. Johannes Rohen hat sich für Anatomie habilitiert.

France - France - Frankreich

Marseille: Le Docteur Marc Romieu, Professeur titulaire de la chaire d'Histo-logie de la Faculté de Médecine, est décédé le 16 juin 1953 dans sa 65e année. 